\title{
Light Mesons and Strange Particle Production at HERA
}

\author{
Leszek Zawiejski* \\ Institute of Nuclear Physics, \\ 152 Radzikowskiego St., \\ 31-342 Kraków, Poland \\ E-mail: Leszek.Zawiejski@ifj.edu.pl \\ On behalf of the ZEUS and HI Collaborations
}

\begin{abstract}
Results on the $\rho^{0}(770), K^{* 0}(892), \phi(1020), K_{S}^{0}$ and $\Lambda$ production in $e p$ collisions at HERA obtained with H1 and ZEUS detectors are presented. Properties of the light mesons are investigated using photoproduction events and those of the strange particles are studied using the deepinelastic scattering events. Photoproduction of light mesons, $\rho^{0}, K^{* 0}$ and $\phi$, at the average $\gamma p$ centre-of-mass energy of $210 \mathrm{GeV}$ and in particular the transverse momentum spectra are studied by H1 Collaboration. The $K_{S}^{0}$ and $\Lambda$ production is evaluated in different regions of the exchanged photon virtuality, $Q^{2}$. Low $2<Q^{2}<100 \mathrm{GeV}^{2}$ and high $145<Q^{2}<20000 \mathrm{GeV}^{2}$ regions are investigated by $\mathrm{H} 1$, whereas the $10<Q^{2}<40000 \mathrm{GeV}^{2}$ region by ZEUS experiment. The differential cross sections for $K_{S}^{0}$ and $\Lambda$ production measured by $\mathrm{H} 1$ in the laboratory and Breit frames are compared to the leading order Monte Carlo predictions. Results of the ZEUS Collaboration studies of the scaled momentum distributions of $K_{S}^{0}$ and $\Lambda$ particles in the Breit frame current fragmentation region in the context of the hadronisation investigation in ep collisions are presented. The data are compared to the leading-logarithm parton-shower Monte Carlo models and next-to-leading order QCD calculations.
\end{abstract}

35th International Conference of High Energy Physics - ICHEP2010,

July 22-28, 2010

Paris France

${ }^{*}$ Speaker. 


\section{Introduction}

Particle production at the HERA collider is the effect of a virtual photon and a proton interactions, $\gamma^{*} p$. Depending on the photon virtuality value, $Q^{2}$, the electron-proton interactions can be separated into a quasi-real photon-proton collisions - photoproduction, with $Q^{2} \approx 0$, and a deepinelastic scattering - DIS, where $Q^{2}>0$. Recently, several aspects of the hadronisation process for these types of $e p$ interactions have been investigated by $\mathrm{H} 1$ and ZEUS experiments. The transverse momentum, the rapidity and the scaled momentum distributions of $\rho^{0}, K^{* 0}$ and $\phi$ mesons (photoproduction) and of strange $K_{S}^{0}$ mesons and $\Lambda$ baryons (DIS) were measured and compared to the predictions of hadroproduction models or next-to-leading order QCD calculations.

\section{Photoproduction of Light Mesons}

In this study only one aspect of the light meson photoproduction studied by H1 Collab. is presented. The measured differential cross sections of the $\rho^{0}, K^{* 0}$ and $\phi$ mesons as a function of their transverse momentum are compared to analogous distributions for other neutral and charged mesons and hadrons in Figure 1. The measured spectra are well described by a power law. The

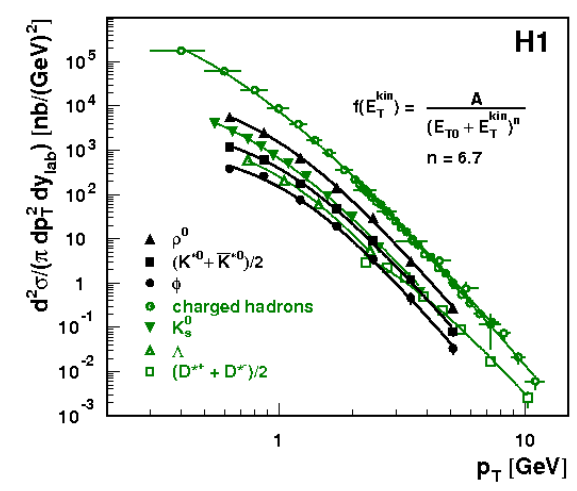

Figure 1: The inclusive cross sections of the light mesons and other particles as a function of the transverse momentum. The curves show the results of fits to a power law cross section parameterisation.

behaviour where particles with different masses, lifetimes and strangeness content are produced with about similar average transverse kinetic energy supports the thermodynamic picture of hadron interactions [1]. Further details of the H1 analysis can be found in [2].

\section{3. $K_{S}^{0}$ and $\Lambda$ DIS Production at Low and High $Q^{2}$}

Strange hadron production, particularly that of baryons, has not been understood yet. Recent H1 and ZEUS results obtained for low and high $Q^{2}$ values allow to extend our knowledge on the fragmentation and hadronisation processes, including strangeness production. Extensive studies on several aspects of $K_{S}^{0}$ and $\Lambda$ production were done by H1 Collab. in the laboratory and Breit frames [3]. The differential cross sections as functions of the photon virtuality, $Q^{2}$, the transverse momentum, $p_{T}$, the pseudorapidity, $\eta$, and Bjorken scaling variable, $x$, were measured and the 
selected results are presented below. The $K_{S}^{0}$ and $\Lambda p_{T}$ spectra and the ratio of the differential cross section for $\Lambda$ production to that of $K_{S}^{0}$ as a function of $\eta$ measured by H1 Collab. for $2<Q^{2}<100$ $\mathrm{GeV}^{2}$ are shown in Figure 2. The distributions were compared to the predictions of the Monte
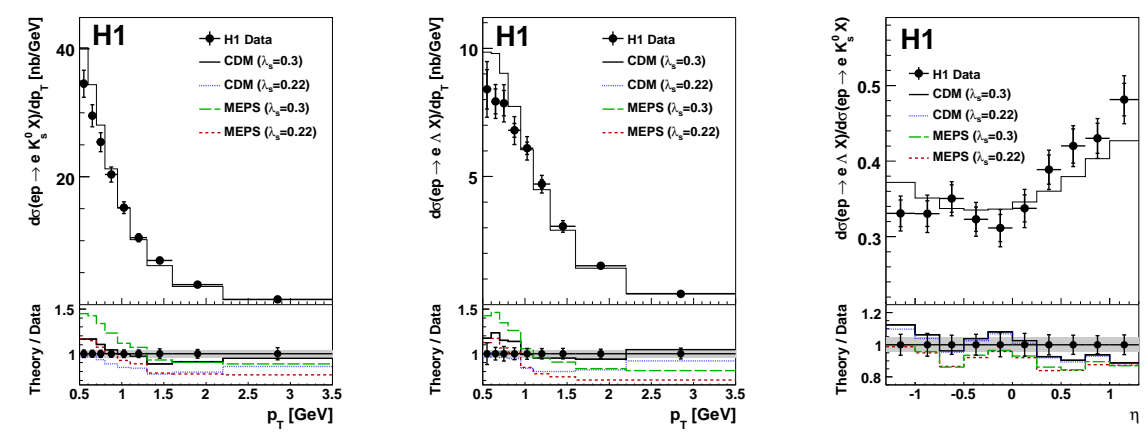

Figure 2: The $p_{T}$ dependence of the production cross sections for $K_{S}^{0}$ and $\Lambda$ particles. The rightmost plot shows the ratio of the differential cross sections for $\Lambda$ baryons and $K_{S}^{0}$ mesons as a function of pseudorapidity.

Carlo models which use different mechanisms for the parton shower development: ARIADNE [4] (referred to as CDM [5]) and LEPTO [6] (referred to as MEPS), but with the same Lund colour string fragmentation model [7] as implemented in the JETSET [8]. For both MC models the strangeness suppression factor $\lambda_{s}$ was set to 0.22 or 0.3 . Other JETSET parameters relating to the strange quark production were set to the values used by ALEPH Collab. The main idea behind $\mathrm{H} 1$ studies was to check if the strange particle production in ep DIS interactions could also be described by the MC models by tuning only the $\lambda_{s}$ parameter. The results obtained for $K_{S}^{0}$ and $\Lambda$ particles indicate that CDM and LEPTO cannot deliver a proper description of the $p_{T}$ spectra in the whole region via tuning of the $\lambda_{s}$ parameter only. The CDM with $\lambda_{s}=0.3$ gives a better description for both $K_{S}^{0}$ and $\Lambda$ data but it overestimates the data in small $p_{T}$ region. The ratio of the differential cross sections for the $\Lambda$ and $K_{S}^{0}$ production, the rightmost plot of Fig. 2, for which a reduction of the theoretical uncertainties was expected, is practically insensitive to the $\lambda_{s}$ changes. The MEPS and CDM models provide a reasonable description of the data only in selected regions of negative $\eta$. For positive $\eta$ both models underestimate the data. A better description of the data by MC predictions was observed for $145<Q^{2}<20000 \mathrm{GeV}^{2}$ [9]. The leftmost plot of Fig. 3 shows the laboratory frame distribution of the $K_{S}^{0}$ transverse momentum. The data are compared with predictions of MEPS and CDM models for two values of $\lambda_{s}=0.22,0.286$. The former value was used in studies in low $Q^{2}$ region. The latter is identical to the one used by ALEPH in their studies of the strange particles in $e^{+} e^{-}$annihilation at LEP. An agreement between the data and MEPS and CDM predictions was found for $\lambda_{s} 0.286$. Additional $\mathrm{H} 1$ studies of $K_{S}^{0}$ and $\Lambda$ production performed in the Breit frame allow for a separation of a struck quark (the current hemisphere) from the proton remnant fragments (the target hemisphere). In Figure 3 the $p_{T}$ distributions are shown separately for the target and current regions (the two right plots). The measured cross section in the target region is about one order of magnitude larger than that measured in the current region. The target hemisphere $p_{T}$ distribution cannot be described by the MEPS or CDM predictions using only one parameter $\lambda_{s}$. The current region is less sensitive to the $\lambda_{s}$ value selection but has much smaller statistics. 

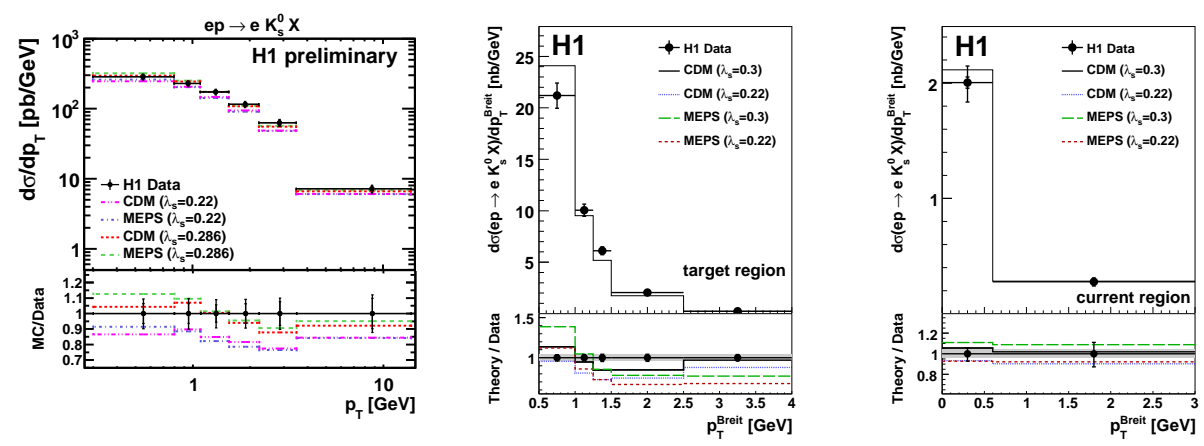

Figure 3: The comparison of the $p_{T}$ distribution for $K_{S}^{0}$ as measured for $145<Q^{2}<20000 \mathrm{GeV}^{2}$ with the MEPS and CDM predictions for different values of the JETSET $\lambda_{s}$. The last two plots show the $p_{T}$ distributions obtained in the Breit frame (target and current hemispheres) for $2<Q^{2}<100 \mathrm{GeV}^{2}$.

\section{4. $K_{S}^{0}$ and $\Lambda$ Scaled Momentum Distributions}

ZEUS Collab. investigated production of $K_{S}^{0}$ and $\Lambda$ in the Breit frame current fragmentation region. The aim was to check the universality of the quark fragmentation function and the factorisation theorem approach used to predict the hadron production in different processes. The scaled momentum distributions were compared with next-to-leading order (NLO) QCD calculations and the leading-log parton-shower MC ARIADNE and LEPTO. Various fragmentation functions (FFs) obtained from fits to $e^{+} e^{-}$data [10,11], to $e^{+} e^{-}$and $p p / p \bar{p}$ data [12], or to $e^{+} e^{-}, p / p \bar{p}$ and $e p$ data [13] were used in the NLO QCD calculations. Figure 4 shows the scaled momentum distributions, $x_{p}=2 P^{\text {Breit }} / Q$, as a function of $Q^{2}$ in different regions of $x_{p}$ for $K_{S}^{0}$ and $\Lambda$ particles. With increasing $Q$ value the phase space for a soft gluon radiation gets enlarged. This leads to
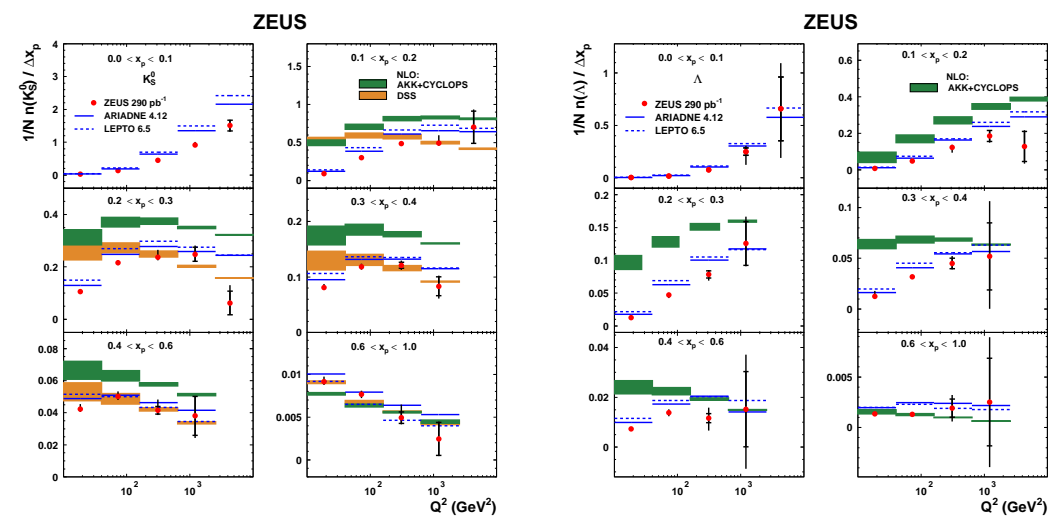

Figure 4: Left: The scaled momentum distributions as function of $Q^{2}$ in different regions of $x_{p}$ for $K_{S}^{0}$ particles together with the predictions of NLO QCD and LO QCD models implemented in MC programs. Right: The corresponding scaled momentum distributions for $\Lambda$ particles.

the rise in the number of soft particles (small $x_{p}$ ) and to the decrease in the number of those with high $x_{p}$. This scaling violation can be seen for $K_{S}^{0}$ and $\Lambda$ particles in Fig. 4 for large $Q^{2}$ values. For $K_{S}^{0}$ the QCD NLO predictions using different FFs describe the data only in certain regions of 
the phase space. For the AKK+CYCLOPS calculations it is limited to $0.6<x_{p}<1$, whereas the DSS calculations describe the data adequately, except for low $x_{p}$ and $Q^{2}$. The ARIADNE 4.12 and LEPTO 6.5 predictions give a good description of the data in almost whole phase space. A similar behaviour is also observed for $\Lambda$ particles in Fig. 4 (right). The data are reasonably well described by the AKK+CYCLOPS calculations only for $0.6<x_{p}<1$. Again MC codes give a better data description.

\section{Summary and Conclusions}

Production of the $\rho^{0}, K^{* 0}, \phi, K_{S}^{0}$ and $\Lambda$ particles has been studied in the photoproduction and deep-inelastic scattering processes at HERA. The $p_{T}$ distributions of the $\rho^{0}, K^{* 0}$ and $\phi$ mesons can be described by a power law distribution. These particles are produced with about the same value of the average transverse kinetic energy which supports a thermodynamic picture of hadron interactions. The differential cross sections as a function of $p_{T}$ and $\eta$, for $K_{S}^{0}$ and $\Lambda$ particles have been measured in low and high $Q^{2}$ regions in the laboratory and Breit frames. The predictions based on the CDM or MEPS models with JETSET selected value of $\lambda_{s}=0.22$ or 0.30 fail to describe the details of the data for $2<Q^{2}<100 \mathrm{GeV}^{2}$ region. For $145<Q^{2}<20000 \mathrm{GeV}^{2}$ region a reasonable agreement with the data has been found for $\lambda_{s}=0.286$. The scaling violation is clearly observed for both $K_{S}^{0}$ and $\Lambda$ particles. Next-to-leading order QCD calculations based on different fragmentation functions can describe the data only in selected regions of $x_{p}$. A better description of the data in most parts of the phase space was supplied by the predictions based on the LO models as implemented into ARIADNE or LEPTO programs.

\section{References}

[1] R. Hagedorn, Nuovo Cimento Suppl. 3 (1965) 147.

[2] H1 Collaboration, F.D. Aaron et al., Phys. Lett. B673 (2009) 119.

[3] H1 Collaboration, F.D. Aaron et al., Eur. Phys. J. C61 (2009) 185.

[4] L. Lönnblad, Comput. Phys. Commun. 71 (1992) 15.

[5] B. Andersson et al., Z. Phys. C43 (1989) 625, L. Lönmblad, Z. Phys. C65 (1995) 285.

[6] G. Ingelman et al., Comput. Phys. Commun. 101 (1997) 108.

[7] T. Sjöstrand, Comp. Phys. Commun. 39 (1986) 347, T. Sjöstrand, M. Bengtsson, Comput. Phys. Commun. 43 (1987) 367, B Andersson et al., Phys. Rep. 97 (1983) 32.

[8] T. Sjöstrand, Comput. Phys. Commun. bf 82 (994) 74.

[9] J. R. Tabasco, Strangeness Production at High $Q^{2}$ at H1, presented at DIS 2010, Florence, Italy.

[10] B.A Kniehl, G. Kramer and B. Pötter, Phys. Rev. Lett. 85 (2000) 5288.

[11] S. Albino, B.A Kniehl, G.Kramer and C. Sandoval, Phys. Rev. D75 (2007) 034018.

[12] S. Albino, B.A. Kniehl and G. Kramer, Nucl. Phys. B803 (2008) 42.

[13] D. de Florian, R. Sassot and M. Stratmann, Phys. Rev. D75 (2007) 114010

D. de Florian, R. Sassot and M. Stratmann, Phys. Rev. D76 (2007) 074033. 